\title{
Feedback Control of Gas Chemistry Reactions in the FIB
}

\author{
L. Zaykova-Feldman and T.M. Moore
}

Omniprobe, Inc., 10410 Miller Rd., Dallas, TX 75238

Gas chemistries in the Focused Ion Beam (FIB) microscope play an important role in semiconductor metrology and process control. Patterned ion and electron beam-assisted chemical vapor deposition (CVD) of materials and etch removal of material have made the FIB an efficient platform for design-edit, mask revision verification, fault isolation, failure analysis and materials analysis. In the FIB in-situ lift-out process, gas-assisted etching speeds the milling process. Beam-assisted CVD in the FIB is important for attachment of lift-out samples to the probe tip and TEM sample holder, for the deposition of protective layers on the surface prior to TEM lift-out, and for the deposition of gap-fill materials, such as silicon dioxide, so that inspected wafers can be returned to the process flow [1,2]. There is a need for accurate and rapid feedback control of these etch and deposition processes to enable high-throughput TEM sample preparation in the FIB. There is also a need for more efficient use of existing ports on commercial FIB vacuum chambers due to the variety of gas chemistries in use and competition for port locations from other accessories such as optical microscopes and x-ray detectors.

Gas injection systems mounted on the wall of the FIB vacuum chamber have become preferred, especially for whole-wafer inspection. A novel multi-source gas injection system with feedback control is shown in Fig. 2. The operational flowchart of this system is shown in Fig. 1. This system contains three crucibles, a carrier/purge gas, feedback from the vacuum chamber, and programmable process flows ("recipes"). The individual sources are maintained at different temperatures to maintain the desired vapor pressure in each tube, and computer-controlled metering valves determine the flow from each crucible and a flow of the carrier/purge gas. The source gasses from the transfer tubes are combined in the final mixing chamber before presenting the combination to the sample surface through a single injection tube. An example of via obtained using an automated etch and deposition routine with this system is shown in Fig.3. The importance of using the carrier or purge gas to enhance the deposition or etch rate has been widely discussed.[3,4]

The first level of feedback is sensitive pressure detection within the gas mixing chamber. The second level of feedback involves detecting the byproducts of the beam-assisted chemical reactions in the FIB vacuum chamber. Combined feedback signals are monitored to adjust the flow rates of the source gases and carrier gas. A Residual Gas Analyzer (RGA) is used to monitor reaction byproducts. The RGA consists of an ionizer, quadrupole mass filter and a detector. Spectra of the residual components in the vacuum chamber are gathered by the RGA and compared with reference spectra of known beamassisted reactions in the FIB. From this comparison the relative performance of the reaction is determined, and adjustments to the flow rates and composition of the combined source gas are made. 


\section{References}

[1] F.A.Stevie et al. Focused Ion Beam Gases for Deposition and Enhanced Etch, in Introduction to Focused Ion Beams: Instrumentation, Theory, Techniques, and Practice, L.A. Giannuzzi, F.A. Stevie (eds), Springer-Verlag New York, 53 (2004).

[2] A.D.D. Ratta et al., J. Vac. Sci. Technol. B11, 2195 (1993).

[3] D.M. Thaus et al., J. Vac. Sci. Technol. B14, 3928 (1996).

[4] J.R. Casey Jr., J. Vac. Sci. Technol. B20, 2682 (2002).

[5] The authors wish to thank Rocky Kruger and Aaron Smith for their dedicated efforts.

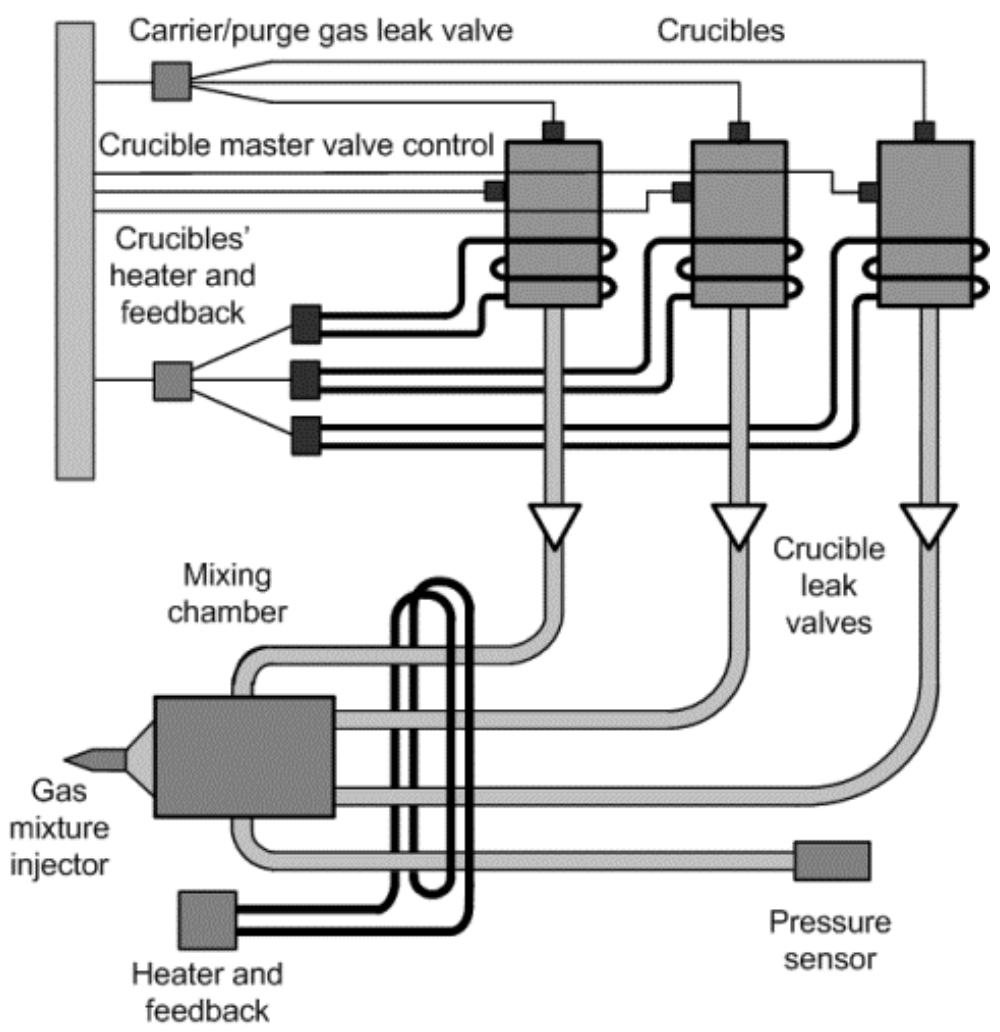

Fig.1. The flowchart of OmniGISTM operation.

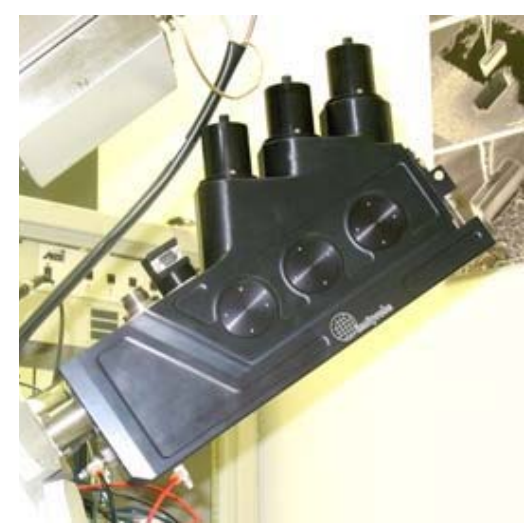

Fig.2. The overall view of OmniGISTM.

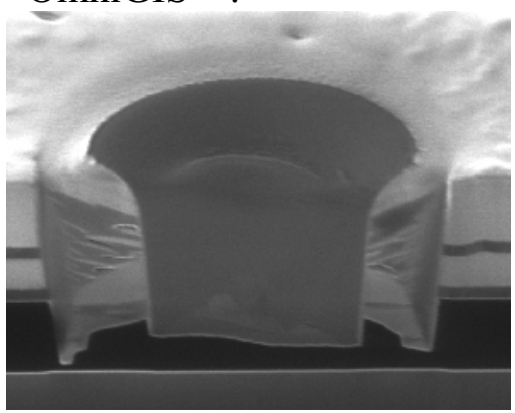

Fig.3. The via obtained using OmniGISTM. 